\title{
De la vie
}

aquatique

à la vie terrestre

Pierre Corvol, Jean-Luc Elghozi

Simone Gilgenkrantz

Il y a 500 millions d'années - longtemps après l'apparition des premiers organismes vivants monocellulaires - des vertébrés tétrapodes, nos lointains ancêtres, vivaient dans les océans. Ces animaux aquatiques, au cours d'une lente transmigration, sont sortis des eaux. Est-ce la mémoire de ce long séjour en milieu liquide qui relie les hommes si étroitement à ce premier des quatre éléments?

Dans la Genèse : «Le souffle de Dieu planait à la surface des eaux », la mythologie: Aphrodite «née de l'écume de la mer », le Veda : «les eaux sont nos mères », I'eau est l'élément primordial. Il en va de même en biologie: l'enfant dans la poche amniotique avant de naître, la composition saline des liquides extracellulaires et la complexité de leur régulation nous rappellent notre origine aquatique.

C'est pourquoi Pierre Corvol et Jean Luc Elghozi ont eu l'idée de choisir pour thème du séminaire de la chaire de médecine expérimentale du Collège de France, en avril 2011, ce sujet: Sortir de l'eau ou le passage de la vie aquatique à la vie terrestre [1]. Aussitôt, on imagine un amphibien - disons la grenouille - qui, après avoir été larve, têtard à longue queue, à deux pattes puis à quatre, enfin devenue anoure, se met à sauter hors du ruisseau d'eau douce.

Mais les choses ne se sont pas passées ainsi. Depuis Darwin, nous savons que l'évolution est lente. Issus des gnathostomes, les sarcoptérigiens aux nageoires paires vont acquérir des pattes marcheuses et devenir des tétrapodes dont certains vont «sortir de l'eau ».

Onze thèmes ont été abordés au cours de ce séminaire.

- Armand de Ricqlès décrit ce processus qui aboutit aux animaux terrestres mais aussi aux poissons

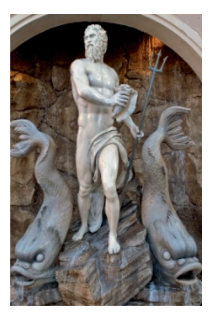

\author{
S. Gilgenkrantz : \\ 9 , rue Basse, \\ 54330 Clérey-sur-Brénon France \\ simsimone.gilgenkrantz@gmail.com
}

d'aujourd'hui, qui, bien que vivant en milieu aquatique, n'ont rien à envier à l'homme en termes d'évolution.

- Milieu aquatique marin ou eau douce? Les poissons euryhalins, ceux qui vont et viennent de l'eau douce à l'eau de mer - comme le saumon ou l'anguille - sont confrontés à d'importants problèmes d'osmorégulation. Les mécanismes physiologiques qui les maintiennent en équilibre, rapportés par Jean-Claude Le Mével de Brest, éclairent certains mécanismes des maladies rénales et cardiovasculaires humaines
Pierre Corvol Jean-Luc Elghozi

\section{Sortir de l'eau}

De la vie aquatique à la vie terrestre
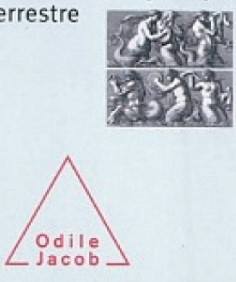

- Une fois sortis de l'eau, pour l'adaptation à la vie terrestre, la mesure de la gravité, la coordination motrice de la marche et l'orientation spatiale nécessitent de subtils organes sensoriels, comme ce précieux système vestibulaire dont les fonctions sont décrites par un groupe de cinq chercheurs ${ }^{1}$.

- De même, avec le passage à la station debout, pour compenser la gravité, un baroréflexe très élaboré intervient et régule la pression artérielle, comme l'explique JeanLuc Elghozi, même dans le cerveau haut perché de la girafe ou du girafon.

- Pierre Corvol reprend ensuite la notion de «milieu intérieur », l'origine de ce concept, pour introduire l'étude des aquaporines, ces «portes de l'eau », présentées par Gabrielle Planelles, puis celles de l'économie du sel, son usage et ses dangers, que Joël Ménard développe dans l'hypertension et les maladies cardio-vasculaires.

${ }^{1}$ A. Berthoz, R. Allain, D. Bennequin, R. David et P. Janvier. 
- En abordant la vie sur terre, les tétrapodes, hors de l'eau, ont parfois été confrontés à des conditions extrêmes: I'endurance à l'effort, le jeûne, le chaud, le froid (Charles Yannick Guezennec) ; ou encore la haute altitude, I'hypoxie et les moyens d'adaptation à celles-ci, ainsi que les pathologies qui en découlent (Jean Paul Richalet).

- Sortir de l'eau ? Certes. Mais ensuite, pourquoi ne pas vouloir y rentrer? La considérer comme bénéfique ? Nicolas Postel Vinay évoque avec érudition la médecine de l'eau, l'histoire de l'hydrothérapie et du thermalisme.

- Enfin, pour prendre un peu de recul, Anne Fagot-Largeault se tourne vers la littérature : les «Louanges de l'eau » de Paul Valéry. Et vers la psychanalyse avec Jung: «les Métamorphoses de l'âme », qui s'opposent à la vision de Bachelard : "L'eau et les rêves » - eaux purificatrices du baptême, eaux violentes du déluge - dont les hommes auraient une approche sensorielle.

Souvent, les livres issus de séminaires manquent d'homogénéité ou de style, les textes étant directement repris de la présentation orale. «Sortir de l'eau » est un vrai livre : chaque chapitre est « écrit » par son auteur. II contient des illustrations qu'on aurait aimé plus abondantes. Milieu marin, eau douce et milieu intérieur, il rassemble tout ce qu'il faut savoir sur le passage de la vie aquatique à la vie terrestre et sur la régulation du milieu intérieur. II a indiscutablement sa place dans la bibliothèque de tout scientifique. $\diamond$ Out of the water or the passage of aquatic life to terrestrial life

\section{RÉFÉRENCE}

1. Corvol P, Elghozi JL (sous la direction de). Sortir de l'eau ou le passage de la vie aquatique à la vie terrestre. Paris : Odile Jacob, 2011 : 240 p.

\section{CONFLIT D'INTÉRÊTS}

L'auteur déclare n'avoir aucun conflit d'intérêts concernant les données publiées dans cet article.

\section{TIRÉS À PART}

S. Gilgenkrantz

\section{Collection SCIENCE ET BIOMÉDECINE}

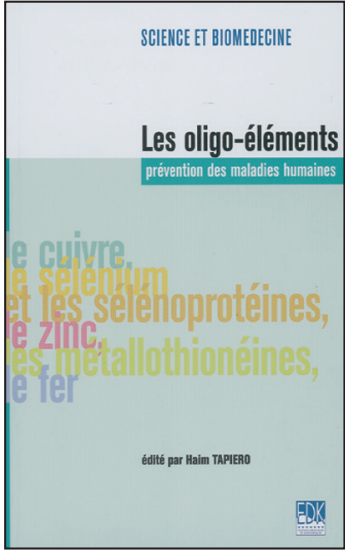

ISBN : 2-84254-107-3 64 pages

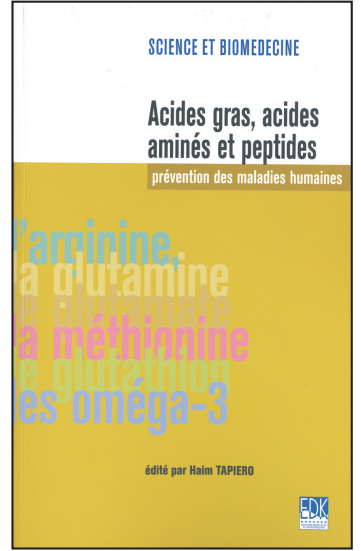

ISBN : 2-84254-108-1 80 pages

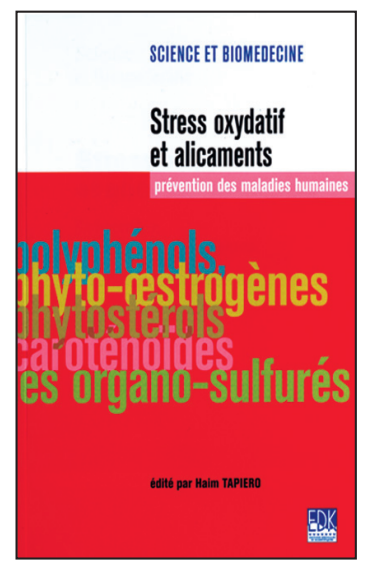

ISBN : 2-84254-111-1 86 pages

\section{Bon de commande}

À retourner à EDK, 25, rue Daviel - 75013 Paris

Tél. : 0158101905 - Fax : 0143293262 - E-mail : edk@edk.fr

NOM : Prénom :

Adresse :

Code postal :

Ville :

Pays :

Fonction :

Je souhaite recevoir l'ouvrage Les oligo-éléments : $10 €+3 €$ de port $=\mathbf{1 3} €$ TTC

Je souhaite recevoir l'ouvrage Acides gras, acides aminés et peptides : $12 €+3 €$ de port $=15 €$ TTC

Je souhaite recevoir l'ouvrage Stress oxydatif et alicaments : $14 €+3 €$ de port $=17 €$ TTC

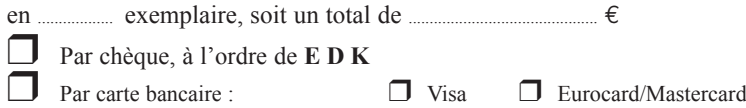

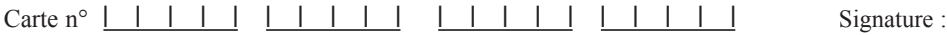

Date d'expiration: $\quad \underline{\mid}|\quad| \quad \mid$

$\mathrm{N}^{\circ}$ de contrôle au dos de la carte : $\quad$ | $\quad|\quad|$ 